ANALYSIS \& PDE Volume $5 \quad$ No. $5 \quad 2012$

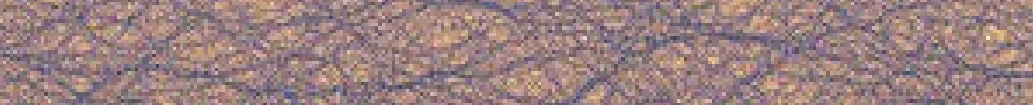
REMI CARLES

NONLINEAR SCHRÖDINGER EQUATION AND FREQUENCY SATURATION 


\title{
NONLINEAR SCHRÖDINGER EQUATION AND FREQUENCY SATURATION
}

\author{
RÉMI CARLES
}

\begin{abstract}
We propose an approach that permits to avoid instability phenomena for the nonlinear Schrödinger equations. We show that by approximating the solution in a suitable way, relying on a frequency cut-off, global well-posedness is obtained in any Sobolev space with nonnegative regularity. The error between the exact solution and its approximation can be measured according to the regularity of the exact solution, with different accuracy according to the cases considered.
\end{abstract}

\section{Introduction}

We consider the nonlinear Schrödinger equation

$$
i \partial_{t} u+\Delta u=\epsilon|u|^{2 \sigma} u, \quad(t, x) \in I \times \mathbb{R}^{d}, \quad u_{\mid t=0}=u_{0},
$$

for some time interval $I \ni 0$, with $\epsilon=1$ (defocusing case) or $\epsilon=-1$ (focusing case). The aim of this paper is to propose an approach to overcome the lack of local well-posedness in Sobolev spaces with nonnegative regularity.

Recall two important invariances associated to (1-1):

- Scaling: if $u$ solves (1-1), then for $\lambda>0$, so does $u_{\lambda}(t, x):=\lambda^{1 / \sigma} u\left(\lambda^{2} t, \lambda x\right)$. This scaling leaves the $\dot{H}_{x}^{s_{c}}$-norm invariant, with $s_{c}=d / 2-1 / \sigma$.

- Galilean: if $u$ solves (1-1), then for $v \in \mathbb{R}^{d}$, so does $e^{i v \cdot x-i|v|^{2} t / 2} u(t, x-v t)$. This transform leaves the $L_{x}^{2}$-norm invariant.

These two arguments suggest that the critical Sobolev regularity to solve (1-1) is $\max \left(s_{c}, 0\right)$. Indeed, if $s_{c} \geqslant 0$, local well-posedness from $H^{s}\left(\mathbb{R}^{d}\right)$ to $H^{s}\left(\mathbb{R}^{d}\right)$ for $s \geqslant s_{c}$ has been established in [Cazenave and Weissler 1990], and if $s_{c}<0$, local well-posedness from $H^{s}\left(\mathbb{R}^{d}\right)$ to $H^{s}\left(\mathbb{R}^{d}\right)$ for $s \geqslant 0$ has been established in [Tsutsumi 1987].

If $s_{c}>0$, pathological phenomena have been exhibited for initial data in $H^{s}\left(\mathbb{R}^{d}\right)$ with $0<s<s_{c}$; Gilles Lebeau has proved a "norm inflation" phenomenon for the wave equation $\partial_{t}^{2} u-\Delta u+u^{p}=0$, $x \in \mathbb{R}^{3}, p \in 2 \mathbb{N}+1, p \geqslant 7$ [Lebeau 2001; Métivier 2004]. The analogous result for (1-1) is this:

This work was supported by the French ANR project R.A.S. number ANR-08-JCJC-0124-01.

MSC2010: primary 35Q55; secondary 35A01, 35B30, 35B45, 35B65.

Keywords: nonlinear Schrödinger equation, well-posedness, approximation. 
Theorem 1.1 [Christ et al. 2003; Burq et al. 2005]. Let $\sigma \geqslant 1$. Assume that $s_{c}=d / 2-1 / \sigma>0$, and let $0<s<s_{c}$. There exists a family $\left(u_{0}^{h}\right)_{0<h \leqslant 1}$ in $\mathscr{Y}\left(\mathbb{R}^{d}\right)$ with

$$
\left\|u_{0}^{h}\right\|_{H^{s}\left(\mathbb{R}^{d}\right)} \rightarrow 0 \quad \text { as } h \rightarrow 0
$$

a solution $u^{h}$ to $(1-1)$ and $0<t^{h} \rightarrow 0$, such that

$$
\left\|u^{h}\left(t^{h}\right)\right\|_{H^{s}\left(\mathbb{R}^{d}\right)} \rightarrow+\infty \quad \text { as } h \rightarrow 0
$$

The argument of the proof consists in considering concentrated initial data

$$
u_{0}(x)=h^{s-d / 2}(\log 1 / h)^{-\alpha} a_{0}\left(\frac{x}{h}\right), \quad \text { with } h \rightarrow 0,
$$

and showing that for very short time, the Laplacian can be neglected in (1-1). The above result then stems from its (easy) counterpart in the ODE case, by choosing a suitable $\alpha>0$. In the spirit of [Lebeau 2005], the above result has been strengthened to a "loss of regularity" in [Alazard and Carles 2009; Carles 2007; Thomann 2008]. The assumptions and conclusion are similar to that in Theorem 1.1; the only difference is that $u^{h}\left(t^{h}, \cdot\right)$ is measured in $H^{k}\left(\mathbb{R}^{d}\right)$ for any $k>s /\left(1+\sigma\left(s_{c}-s\right)\right)$, so $k$ is allowed to be smaller than $s$. In all the cases mentioned here, the lack of uniform continuity of the nonlinear flow map near the origin is due to the appearance of higher and higher frequencies on a very short time scale. If $s_{c}<0$, similar pathological phenomena have been established in $H^{s}\left(\mathbb{R}^{d}\right)$ with $s<0$, where on the contrary, low frequencies are ignited; see [Bejenaru and Tao 2006; Carles et al. 2012; Christ et al. 2003; Kenig et al. 2001]. In the rest of this paper, we focus on nonnegative regularity, $s \geqslant 0$.

The goal of this paper is twofold. First, we want to investigate a method to remove the pathology mentioned above, causing a lack of well-posedness for (1-1), in a deterministic way, as opposed to the probabilistic approach initiated in [Burq and Tzvetkov 2008a; 2008b] for the wave equation. The other motivation is related to numerical simulations for (1-1), where high frequencies may be a source of important errors; see for instance [Ignat and Zuazua 2012], a reference which will be discussed in further detail in Sections 3 and 4.

We show that with a suitable cut-off on the high frequencies of the nonlinearity, the obstructions to local well-posedness vanish, and the problem becomes globally well-posed: the nonlinear evolution of any initial datum in $L^{2}\left(\mathbb{R}^{d}\right)$ can be controlled a priori, an information which may be useful for numerics, since we do not have to decide if the initial datum belongs to a full measure set or not. This strategy is validated inasmuch as this procedure yields a good approximation of the solution to (1-1) as the cut-off tends to the identity. Note that this approach can be viewed as a deterministic counterpart of the one presented in [Burq et al. 2012] (see also [Burq 2011]). There, for the one-dimensional $L^{2}$-supercritical defocusing nonlinear Schrödinger equation, the authors construct a Gibbs measure such that, among other features, the pathological phenomenon described in Theorem 1.1 occurs for a set of initial data whose measure is zero: on the support of the Gibbs measure, the Cauchy problem is globally well-posed, and a scattering theory is available. Both points of view aim at showing that norm inflation in the sense of Theorem 1.1 is a rare phenomenon: in [Burq et al. 2012], the authors give a rigorous meaning to this 
statement in an abstract way, while we are rather interested in a recipe to avoid instabilities for sure, by a suitable approximation of the equation, which can be used typically for numerical simulations.

Our choice of cutting off the high frequencies instead of, for instance, the values of the function itself is indeed motivated by numerics, where it is standard to filter out high frequencies (sometimes without even saying so). In an appendix, we discuss another approach, consisting in saturating the values of the nonlinearity. One could of course combine both approaches, frequency and physical saturations.

Notation. We define the Fourier transform by the formula

$$
\widehat{f}(\xi)=\mathscr{F}(f)(\xi)=\frac{1}{(2 \pi)^{d / 2}} \int_{\mathbb{R}^{d}} e^{-i x \cdot \xi} f(x) d x, \quad f \in \mathscr{Y}\left(\mathbb{R}^{d}\right) .
$$

We write $a \lesssim b$ if there exists $C$ such that $a \leqslant C b$. In the presence of a small parameter $h$, the notation indicates that $C$ is independent of $h \in(0,1]$.

\section{From instability to global well-posedness}

Let $\chi: \mathbb{R}^{d} \rightarrow[0,1]$ be a smooth function, equal to one on the unit ball, and even: $\chi(-x)=\chi(x)$ for all $x \in \mathbb{R}^{d}$. It may be compactly supported, in the Schwartz class $\mathscr{Y}\left(\mathbb{R}^{d}\right)$, or with a slower decay at infinity. For simplicity, we will not discuss sharp assumptions on $\chi$. We define the frequency "cut-off" $\Pi$ as the Fourier multiplier

$$
\widehat{\Pi(f)}(\xi)=\chi(\xi) \widehat{f}(\xi)
$$

As pointed out in the introduction, in the examples constructed to prove the lack of local well-posedness, the mechanism of high frequencies amplification occurs at the level of the ordinary differential equation. We discuss some strategies to saturate high frequencies at the ODE level first, with $\epsilon=1$ for simplicity.

2A. Candidates at the ODE level. The first possibility to prevent the appearance of high frequencies by nonlinear self-interaction consists in saturating the whole nonlinearity:

$$
i \partial_{t} v=\Pi\left(|v|^{2 \sigma} v\right)
$$

This can be viewed as an extremely simplified version of the $I$-method (see [Colliander et al. 2002]). Another choice consists in saturating the high frequencies in the "nonlinear multiplicative potential" only, that is $|v|^{2 \sigma}$. For $\sigma \in \mathbb{N}$, we propose two possibilities,

$$
\begin{aligned}
i \partial_{t} v & =\Pi\left(|v|^{2 \sigma}\right) v, \\
i \partial_{t} v & =\left(\Pi\left(|v|^{2}\right)\right)^{\sigma} v .
\end{aligned}
$$

In the cubic case $\sigma=1$, the last two approaches obviously coincide. These approaches have two advantages over (2-1):

- They preserve gauge invariance. If $v$ solves the equation, then so does $v e^{i \theta}$ for any constant $\theta \in \mathbb{R}$.

- They preserve conservation of mass. 
To see the second point, rewrite $\Pi(f)$ as $K * f$, with $K(x)=(2 \pi)^{-d / 2} \widehat{\chi}(-x)$. Since $\chi$ is even and real-valued, so is $K$, and therefore $\partial_{t}|v|^{2}=0$ in (2-2) and (2-3). This identity leads to the conservation of the $L^{2}$-norm at the PDE level.

Before passing to the PDE case, we conclude this section by showing that even at the ODE level, cutting off high frequencies in the initial data does not suffice to prevent the appearance of higher frequencies in the solution for positive time. For $a \in \mathscr{Y}\left(\mathbb{R}^{d}\right)$ and $s>0$, consider the solution $v^{h}$ to

$$
i \partial_{t} v^{h}=\left|v^{h}\right|^{2 \sigma} v^{h}, \quad v^{h}(0, x)=h^{s-d / 2} a\left(\frac{x}{h}\right) .
$$

Then $v^{h}{ }_{\mid t=0}$ is bounded in $H^{s}\left(\mathbb{R}^{d}\right)$, uniformly in $h \in(0,1]$, and if $\widehat{a}$ is compactly supported (in $B(0, R)$ ), then $\widehat{v}_{\mid t=0}$ is compactly supported (in $B(0, R / h)$ ). Since $\partial_{t}\left|v^{h}\right|^{2}=0$, we have the explicit formula

$$
v^{h}(t, x)=h^{s-d / 2} a\left(\frac{x}{h}\right) \exp \left(-i t h^{2 \sigma(s-d / 2)}\left|a\left(\frac{x}{h}\right)\right|^{2 \sigma}\right) .
$$

We check that for $t>0$, as $h \rightarrow 0$, the homogeneous Sobolev norms behave like

$$
\left\|v^{h}(t)\right\|_{\dot{H}^{k}} \approx h^{s-2 k \sigma(s-d / 2)-k} t^{k},
$$

at least for $k \in \mathbb{N}$. The above quantity is unbounded as $h \rightarrow 0$ if

$$
k>\frac{s}{1+2 \sigma(s-d / 2)} \text {. }
$$

Therefore, if $s<d / 2, v^{h}(t, \cdot)$ is unbounded in $H^{s}\left(\mathbb{R}^{d}\right)$ for $t>0$, as $h \rightarrow 0$ : cutting off the high frequencies in the initial data does not suffice to control the frequency support of the solution. On the other hand, the models (2-2) and (2-3) prevent the appearance of high frequencies by nonlinear self-interaction. The above mechanism is essentially the one that leads to the norm inflation phenomenon in [Burq et al. 2005; Christ et al. 2003; Lebeau 2001], except that in those papers, the approximation by an ODE is used only on a time interval where the $H^{s}$-norm becomes unbounded, but not the $H^{k}$-norm for any $k<s$. The above mechanism at the PDE level leads to the loss of regularity [Alazard and Carles 2009; Carles 2007; Lebeau 2005; Thomann 2008], where indeed $k$ is allowed to be smaller than $s$, as recalled in the introduction. Roughly speaking, the appearance of oscillations is quite similar to the above ODE example; in the PDE case, the numerology is different, and the proof is more intricate.

2B. Choice at the PDE level. We consider now the equations

$$
\begin{aligned}
& i \partial_{t} u+P(D) u=\epsilon \Pi\left(|u|^{2 \sigma}\right) u, \\
& i \partial_{t} u+P(D) u=\epsilon\left(\Pi\left(|u|^{2}\right)\right)^{\sigma} u,
\end{aligned}
$$

where $P(D)$ is a Fourier multiplier with a real-valued symbol $P: \mathbb{R}^{d} \rightarrow \mathbb{R}$,

$$
\widehat{P(D) f}=P(\xi) \widehat{f}(\xi) .
$$

The $L^{2}$-norm of $u$ is formally independent of time:

$$
\frac{d}{d t} \int_{\mathbb{R}^{d}}|u(t, x)|^{2} d x=0 .
$$


In view of this conservation and of the Young inequality

$$
\|\Pi(f)\|_{L^{\infty}} \leqslant\|K\|_{L^{\infty}}\|f\|_{L^{1}}
$$

the option (2-5) seems more interesting than (2-4), and we have the following result.

Theorem 2.1. Let $\sigma \in \mathbb{N}, \epsilon \in\{ \pm 1\}, P: \mathbb{R}^{d} \rightarrow \mathbb{R}$ and $\chi \in \mathscr{S}\left(\mathbb{R}^{d}\right)$ even and real-valued.

- For any $u_{0} \in L^{2}\left(\mathbb{R}^{d}\right),(2-5)$ has a unique solution $u \in C\left(\mathbb{R} ; L^{2}\left(\mathbb{R}^{d}\right)\right)$ such that $u_{\mid t=0}=u_{0}$. Its $L^{2}$-norm is independent of time; (2-6) holds.

- If in addition $u_{0} \in H^{s}\left(\mathbb{R}^{d}\right), s \in \mathbb{N}$, then $u \in C\left(\mathbb{R} ; H^{s}\left(\mathbb{R}^{d}\right)\right)$.

- The flow map $u_{0} \mapsto u$ is uniformly continuous from the balls in $L^{2}\left(\mathbb{R}^{d}\right)$ to $C\left(\mathbb{R} ; L^{2}\left(\mathbb{R}^{d}\right)\right)$. More precisely, for all $u_{0}, v_{0} \in L^{2}\left(\mathbb{R}^{d}\right)$, there exists $C$ depending on $\sigma,\|K\|_{L^{\infty}},\left\|u_{0}\right\|_{L^{2}}$ and $\left\|v_{0}\right\|_{L^{2}}$ such that, for all $T>0$,

$$
\|u-v\|_{L^{\infty}\left([-T, T] ; L^{2}\left(\mathbb{R}^{d}\right)\right)} \leqslant\left\|u_{0}-v_{0}\right\|_{L^{2}\left(\mathbb{R}^{d}\right)} e^{C T},
$$

where $u$ and $v$ denote the solutions to (2-5) with initial data $u_{0}$ and $v_{0}$, respectively.

- More generally, let $s \in \mathbb{N}$. For all $u_{0}, v_{0} \in H^{s}\left(\mathbb{R}^{d}\right)$, there exists $C$ depending on $\sigma,\|K\|_{W^{s, \infty}}\left\|u_{0}\right\|_{H^{s}}$ and $\left\|v_{0}\right\|_{H^{s}}$ such that for all $T>0$,

$$
\|u-v\|_{L^{\infty}\left([-T, T] ; H^{s}\left(\mathbb{R}^{d}\right)\right)} \leqslant\left\|u_{0}-v_{0}\right\|_{H^{s}\left(\mathbb{R}^{d}\right)} e^{C T} .
$$

Remark 2.2. As pointed out in [Cazenave et al. 2011], even if the solution is constructed by a fixed point argument, the continuity of the flow map is not trivial in general. In the case of Schrödinger equations (1-1), continuity of the flow map in $H^{s}\left(\mathbb{R}^{d}\right)$ is known only in a limited number of cases: see [Tsutsumi 1987] for $s=0$, [Kato 1987] for $s=1$ and $s=2$, and [Cazenave et al. 2011] for $0<s<1$.

Proof. First, recall that $S(t)=e^{-i t P(D)}$ is a unitary group on $\dot{H}^{s}\left(\mathbb{R}^{d}\right), s \in \mathbb{R}$. Duhamel's formula associated to $(2-5)$ reads

$$
u(t)=S(t) u_{0}-i \epsilon \int_{0}^{t} S(t-\tau)\left(\left(K *|u|^{2}\right)^{\sigma} u\right)(\tau) d \tau .
$$

The local existence in $L^{2}$ stems from a standard fixed point argument in

$$
X(T)=\left\{u \in C\left([-T, T] ; L^{2}\left(\mathbb{R}^{d}\right)\right) \mid\|u\|_{L^{\infty}\left([-T, T] ; L^{2}\right)} \leqslant 2\left\|u_{0}\right\|_{L^{2}}\right\} .
$$

Denote by $\Phi(u)(t)$ the right hand side of (2-10). In view of (2-7), for $t \in[-T, T]$,

$$
\begin{aligned}
\|\Phi(u)(t)\|_{L^{2}} & \leqslant\left\|u_{0}\right\|_{L^{2}}+\int_{-T}\left\|\left(\left(K *|u|^{2}\right)^{\sigma} u\right)(\tau)\right\|_{L^{2}} d \tau \\
& \leqslant\left\|u_{0}\right\|_{L^{2}}+\int_{-T}^{T}\left\|K *|u(\tau)|^{2}\right\|_{L^{\infty}}^{\sigma}\|u(\tau)\|_{L^{2}} d \tau \\
& \leqslant\left\|u_{0}\right\|_{L^{2}}+\|K\|_{L^{\infty}}^{\sigma} \int_{-T}^{T}\|u(\tau)\|_{L^{2}}^{2 \sigma+1} d \tau .
\end{aligned}
$$

By choosing $T>0$ sufficiently small, we see that $X(T)$ is stable under the action of $\Phi$. Note that in the case of the model (2-4), the above estimate would have to be adapted, forcing us to work in a space smaller 
than $X(T)$ ( $L^{2}$ regularity in space would no longer be sufficient in general). Contraction is established in the same way:

$$
\begin{aligned}
\|\Phi(u)(t)-\Phi(v)(t)\|_{L^{2}} & \leqslant \int_{-T}^{T}\left\|\left(\left(K *|u|^{2}\right)^{\sigma} u\right)(\tau)-\left(\left(K *|v|^{2}\right)^{\sigma} v\right)(\tau)\right\|_{L^{2}} d \tau \\
& \leqslant \int_{-T}^{T}\left\|\left(\left(K *|u|^{2}\right)^{\sigma}-\left(K *|v|^{2}\right)^{\sigma}\right) u\right\|_{L^{2}} d \tau+\int_{-T}^{T}\left\|\left(\left(K *|v|^{2}\right)^{\sigma}\right)(u-v)\right\|_{L^{2}} d \tau .
\end{aligned}
$$

Using the estimate $\left|a^{\sigma}-b^{\sigma}\right| \lesssim\left(|a|^{\sigma-1}+|b|^{\sigma-1}\right)|a-b|$, and (2-7) again, we infer $\|\Phi(u)(t)-\Phi(v)(t)\|_{L^{2}}$

$$
\lesssim\|K\|_{L^{\infty}}^{\sigma} \int_{-T}^{T}\left(\|u\|_{L^{2}}^{2 \sigma-1}+\|v\|_{L^{2}}^{2 \sigma-1}\right)\|u-v\|_{L^{2}}\|u\|_{L^{2}} d \tau+\|K\|_{L^{\infty}}^{\sigma} \int_{-T}^{T}\|v\|_{L^{2}}^{2 \sigma}\|u-v\|_{L^{2}} d \tau,
$$

where all the functions inside the integrals are implicitly evaluated at time $\tau$. Choosing $T>0$ possibly smaller, $\Phi$ is a contraction on $X(T)$. Note that this small time $T$ depends only on $\sigma,\|K\|_{L^{\infty}}$ and $\left\|u_{0}\right\|_{L^{2}}$. Since the $L^{2}$-norm of $u$ is preserved (see [Cazenave 2003] for a rigorous justification), the construction of a local solution can be repeated indefinitely, hence global existence and uniqueness at the $L^{2}$ level.

Global existence in $H^{s}\left(\mathbb{R}^{d}\right)$ for $s \in \mathbb{N}$ then follows easily, thanks to the estimate

$$
\left\|\left(K *|u|^{2}\right)^{\sigma} u\right\|_{H^{s}} \lesssim \sum_{|\alpha|+|\beta|=s}\left\|\partial^{\alpha}\left(K *|u|^{2}\right)^{\sigma} \partial^{\beta} u\right\|_{L^{2}} \lesssim\|K\|_{W^{s, \infty}}^{\sigma}\|u\|_{L^{2}}^{\sigma}\|u\|_{H^{s}} .
$$

The continuity of the flow map in $L^{2}$ is obtained by resuming the estimate written to establish the contraction of $\Phi$ : For $t>0$,

$$
\begin{aligned}
\|u(t)-v(t)\|_{L^{2}} & \leqslant\left\|u_{0}-v_{0}\right\|_{L^{2}}+\|K\|_{L^{\infty}}^{\sigma} \int_{0}^{t}\left(\|u\|_{L^{2}}^{2 \sigma}+\|v\|_{L^{2}}^{2 \sigma}\right)\|u-v\|_{L^{2}} d \tau \\
& \leqslant\left\|u_{0}-v_{0}\right\|_{L^{2}}+\|K\|_{L^{\infty}}^{\sigma}\left(\left\|u_{0}\right\|_{L^{2}}^{2 \sigma}+\left\|v_{0}\right\|_{L^{2}}^{2 \sigma}\right) \int_{0}^{t}\|u-v\|_{L^{2}} d \tau,
\end{aligned}
$$

where we have used the conservation of the $L^{2}$-norm. Proceeding similarly for $t<0$, Gronwall's lemma then yields (2-8) for $C$ depending only of $\sigma,\|K\|_{L^{\infty}},\left\|u_{0}\right\|_{L^{2}}$ and $\left\|v_{0}\right\|_{L^{2}}$. Finally, (2-9) is obtained in a similar fashion.

Remark 2.3. The proof of continuity of the flow map is easy. This is in sharp contrast with the case of the equation without frequency cut-off. In the case of Schrödinger equations $\left(P(\xi)=-|\xi|^{2}\right)$, continuity is more intricate to establish (see [Tsutsumi 1987]), and is true only for $L^{2}$-subcritical nonlinearities, $\sigma \leqslant 2 / d$, from [Christ et al. 2003].

We note that even for large $\sigma$, global well-posedness in $L^{2}$ is available, in sharp contrast with the nonlinear Schrödinger equation (1-1). Even in the focusing case $\epsilon=-1$, the high frequency cut-off prevents finite time blow-up. In (2-9), consider $v_{0}=v=0$ and $s=1$ for instance: by comparison with the case of (1-1), we see that the constant $C$ necessarily depends on $K$ (or equivalently on $\chi$ ), and is unbounded as $\chi$ converges to the Dirac mass. The frequency cut-off $\Pi$ removes the instabilities, and prevents finite time blow-up. 
Remark 2.4 (Hamiltonian structure in the cubic case). If $\sigma=1,(2-4)$ and (2-5) coincide. We have the equivalence

$$
\chi \text { even and real-valued } \Longleftrightarrow K \text { even and real-valued. }
$$

This implies that under the assumption of Theorem 2.1, (2-5) has an Hamiltonian structure, and the conserved energy is

$$
H(u)=\int_{\mathbb{R}^{d}} \bar{u}(x) P(D) u(x) d x+\frac{\epsilon}{2} \iint K(x-y)|u(y)|^{2}|u(x)|^{2} d x d y .
$$

\section{Convergence in the smooth case}

Suppose that $P(D)$ converges to $\Delta$ and that $\Pi$ converges to Id, does the solution to (2-5) then converge to the solution of NLS? We show that this is the case under suitable assumptions on these convergences, at least in the case where the solution to the limiting Equation (1-1) is very smooth. In the sequel, the convergence is indexed by $h \in(0,1]$.

Proposition 3.1. Let $\sigma \in \mathbb{N}$. We assume that $P$ and $\Pi$ verify the following properties:

- There exist $\alpha, \beta \geqslant 0$ such that $P_{h}(\xi)=-|\xi|^{2}+\mathcal{O}\left(h^{\alpha}\langle\xi\rangle^{\beta}\right)$.

- $\chi_{h}(\xi)=\chi(h \xi)$, with $\chi \in \mathscr{S}\left(\mathbb{R}^{d} ;[0,1]\right)$ even, real-valued, $\chi=1$ on the unit ball.

Denote by $u^{h}$ the solution to (2-5) with $P_{h}$ and $\chi_{h}$, such that $u_{\mid t=0}^{h}=u_{\mid t=0}$. Suppose that the solution to (1-1) satisfies $u \in L^{\infty}\left([0, T] ; H^{s+\beta}\right)$, for some $s>d / 2$. Then

$$
\left\|u-u^{h}\right\|_{L^{\infty}\left([0, T] ; H^{s}\right)} \lesssim h^{\min (\alpha, \beta)} .
$$

Example 3.2. The above assumption on $P_{h}$ is satisfied with $\alpha=1$ and $\beta=2$ in the following cases:

- $P_{h}(\xi)=\frac{-|\xi|^{2}}{1+h|\xi|^{2}}$.

- $P_{h}(\xi)=-\frac{1}{h} \arctan \left(h|\xi|^{2}\right)$.

The second example is borrowed from [Debussche and Faou 2009], where this truncated operator appears naturally when discretizing the Laplacian for numerical schemes.

Remark 3.3. In this result, no assumption is needed on the possible decay of $\chi$ at infinity.

Proof. Let $w^{h}=u-u^{h}$. It satisfies $w_{\mid t=0}^{h}=0$ and

$$
\begin{aligned}
i \partial_{t} w^{h}+P_{h}(D) w^{h}=\epsilon\left(\Pi_{h}\left(|u|^{2}\right)\right)^{\sigma} u & -\epsilon\left(\Pi_{h}\left(\left|u^{h}\right|^{2}\right)\right)^{\sigma} u^{h} \\
& +\left(P_{h}(D)-\Delta\right) u+\epsilon\left(|u|^{2 \sigma}-\left(\Pi_{h}\left(|u|^{2}\right)\right)^{\sigma}\right) u,
\end{aligned}
$$

where we have denoted by $\Pi_{h}$ the Fourier multiplier of symbol $\chi_{h}$. Denote by $R^{h}(u)$ the second line, which corresponds to a source term. In view of the assumption on $P_{h}$, there exists $C$ independent of $h \in(0,1]$ such that

$$
\left\|P_{h}(D) f-\Delta f\right\|_{H^{s}} \leqslant C h^{\alpha}\|f\|_{H^{s+\beta}} \quad \text { for all } f \in H^{s+\beta}\left(\mathbb{R}^{d}\right) .
$$


We also have, by the Plancherel formula,

$$
\begin{aligned}
\left\|\left(1-\Pi_{h}\right) f\right\|_{H^{s}}^{2} & =\int_{\mathbb{R}^{d}}(1-\chi(h \xi))^{2}\langle\xi\rangle^{2 s}|\widehat{f}(\xi)|^{2} d \xi \\
& \leqslant \int_{|\xi|>1 / h}\langle\xi\rangle^{2 s}|\widehat{f}(\xi)|^{2} d \xi \leqslant h^{2 \beta} \int_{|\xi|>1 / h}\langle\xi\rangle^{2 s+2 \beta}|\widehat{f}(\xi)|^{2} d \xi \leqslant h^{2 \beta}\|f\|_{H^{s+\beta}}^{2} .
\end{aligned}
$$

Therefore,

$$
\left\|R^{h}(u)\right\|_{L^{\infty}\left([0, T] ; H^{s}\right)} \lesssim h^{\min (\alpha, \beta)}\|u\|_{L^{\infty}\left([0, T] ; H^{s+\beta}\right)} .
$$

Now since $s>d / 2, H^{s}\left(\mathbb{R}^{d}\right)$ is an algebra, and there exists $C$ independent of $h$ such that

$$
\left\|\left(\Pi_{h}\left(|u|^{2}\right)\right)^{\sigma} u-\left(\Pi_{h}\left(\left|u^{h}\right|^{2}\right)\right)^{\sigma} u^{h}\right\|_{H^{s}} \leqslant C\|\widehat{\chi}\|_{L^{1}}^{\sigma}\left(\|u\|_{H^{s}}^{2 \sigma}+\left\|u^{h}\right\|_{H^{s}}^{2 \sigma}\right)\left\|u-u^{h}\right\|_{H^{s}},
$$

where the Young inequality that we have used is not the same as in Section 2:

$$
\|K * f\|_{L^{2}} \leqslant\|K\|_{L^{1}}\|f\|_{L^{2}}
$$

This is essentially the only way to obtain an estimate independent of $h \in(0,1]$. Indeed, $\Pi_{h}(f)=K_{h} * f$, with

$$
K_{h}(x)=\frac{1}{(2 \pi)^{d / 2} h^{d}} \widehat{\chi}\left(\frac{-x}{h}\right) .
$$

The result then stems from a bootstrap argument: so long as

$$
\left\|u^{h}\right\|_{L^{\infty}\left([0, t] ; H^{s}\right)} \leqslant 1+\|u\|_{L^{\infty}\left([0, T] ; H^{s}\right)},
$$

Gronwall's lemma yields

$$
\left\|u-u^{h}\right\|_{L^{\infty}\left([0, t] ; H^{s}\right)} \lesssim h^{\min (\alpha, \beta)}\|u\|_{L^{\infty}\left([0, T] ; H^{s+\beta}\right)} .
$$

Therefore, up to choosing $h$ sufficiently small, this estimate is valid up to $t=T$.

Such a convergence result can be compared to the one proved in [Ignat and Zuazua 2012] to prove the convergence of numerical approximations. The approach there is a bit different though, inasmuch as the frequency cut-off does not affect the nonlinearity (as in (2-5)), but the initial data: consider a solution $v^{h}$ to

$$
i \partial_{t} v^{h}+P_{h}(D) v^{h}=\epsilon\left|v^{h}\right|^{2 \sigma} v^{h}, \quad v_{\mid t=0}^{h}=\Pi_{h} u_{0}
$$

Ignat and Zuazua proved that the discrete analogue of $\Pi_{h} u-v_{h}$ is small. Proposition 3.1 differs from their results in several aspects:

- The context in [Ignat and Zuazua 2012] is discrete.

- Only the low frequency part of $u, \Pi_{h} u$, is shown to be well approximated.

- The regularity assumption on $u$ may be much weaker. 
As mentioned above, the second point is due to the choice of the model. However, as discussed in Section 2A, controlling the high frequencies of the initial data must not be expected to ensure a control of high frequencies of the solution $v^{h}$ for positive time.

The third point is due to the use of Strichartz estimates in [Ignat and Zuazua 2012]. In the next section, we show that in the presence of dispersion (with $P_{h}(\xi)=-|\xi|^{2}$ ), Proposition 3.1 can be adapted to rougher data.

\section{Convergence using dispersive estimates}

We first recall a standard definition.

Definition 4.1. A pair $(p, q) \neq(2, \infty)$ is admissible if $p \geqslant 2, q \geqslant 2$, and

$$
\frac{2}{p}=d\left(\frac{1}{2}-\frac{1}{q}\right)
$$

We shall consider (2-5) when $P(D)$ is exactly the Laplacian, and not an approximation as in Proposition 3.1. The reason is that when $P$ is bounded, then no Strichartz estimate is available, as we now recall. Let $S(\cdot)$ be bounded on $H^{s}$ for all $s \geqslant 0$. By the Sobolev embedding, for all $(p, q)$ (not necessarily admissible) with $2 \leqslant q<\infty$, there exists $C>0$ such that for all $u_{0} \in H^{d / 2-d / q}\left(\mathbb{R}^{d}\right)$, and all finite time interval $I$,

$\left\|S(\cdot) u_{0}\right\|_{L^{p}\left(I ; L^{q}\left(\mathbb{R}^{d}\right)\right)} \leqslant C\left\|S(\cdot) u_{0}\right\|_{L^{p}\left(I ; H^{d / 2-d / q}\left(\mathbb{R}^{d}\right)\right)} \leqslant C\left\|u_{0}\right\|_{L^{p}\left(I ; H^{d / 2-d / q}\left(\mathbb{R}^{d}\right)\right)}=C|I|^{1 / p}\left\|u_{0}\right\|_{H^{d / 2-d / q\left(\mathbb{R}^{d}\right)}}$.

If the Fourier multiplier $P$ is bounded, the above estimate cannot be improved, in sharp contrast with the result provided by the Strichartz estimates.

Proposition 4.2 [Carles 2011]. Let $d \geqslant 1$, and $P \in L^{\infty}\left(\mathbb{R}^{d} ; \mathbb{R}\right)$. Write $S(t)=e^{-i t P(D)}$. Suppose that there exist an admissible pair $(p, q)$, an index $k \in \mathbb{R}$, a time interval $I \ni 0,|I|>0$, and a constant $C>0$ such that

$$
\left\|S(\cdot) u_{0}\right\|_{L^{p}\left(I ; L^{q}\left(\mathbb{R}^{d}\right)\right)} \leqslant C\left\|u_{0}\right\|_{H^{k}\left(\mathbb{R}^{d}\right)} \quad \text { for all } u_{0} \in H^{k}\left(\mathbb{R}^{d}\right) .
$$

Then necessarily $k \geqslant 2 / p=d / 2-d / q$.

We now state the main result of this section.

Theorem 4.3. Let $\sigma \in \mathbb{N}$ and $T>0$. We assume that $\chi_{h}(\xi)=\chi(h \xi)$, with $\chi \in \mathscr{Y}\left(\mathbb{R}^{d}\right)$ even, real-valued, $\chi=1$ on $B(0,1)$. Let $u$ solve $(1-1)$, and consider the solution $u^{h}$ to

$$
i \partial_{t} u^{h}+\Delta u^{h}=\epsilon\left(\Pi_{h}\left(\left|u^{h}\right|^{2}\right)\right)^{\sigma} u^{h}, \quad u_{\mid t=0}^{h}=u_{0} .
$$

1. Suppose that $\sigma=1$ and $d \leqslant 2$. If $u \in L^{\infty}\left([0, T] ; L^{2}\right) \cap L^{8 / d}\left([0, T] ; L^{4}\right)$, then

$$
\left\|u-u^{h}\right\|_{L^{\infty}\left([0, T] ; L^{2}\right) \underset{h \rightarrow 0}{\longrightarrow} 0 .} 0 .
$$

2. Suppose that $\sigma=1$ and $d=3$. 
- If $u, \nabla u \in L^{\infty}\left([0, T] ; L^{2}\right) \cap L^{8 / d}\left([0, T] ; L^{4}\right)$, then

$$
\left\|u-u^{h}\right\|_{L^{\infty}\left([0, T] ; H^{1}\right)} \underset{h \rightarrow 0}{\longrightarrow} 0 .
$$

- If $u \in L^{\infty}\left([0, T] ; H^{s}\right)$, with $s>3 / 2$, then

$$
\left\|u-u^{h}\right\|_{L^{\infty}\left([0, T] ; L^{2}\right)} \lesssim h^{s} \quad \text { and } \quad\left\|u-u^{h}\right\|_{L^{\infty}\left([0, T] ; H^{1}\right)} \lesssim h^{s-1} .
$$

3. Suppose that $\sigma \geqslant 1$ and $d \leqslant 2$. If $u \in L^{\infty}\left([0, T] ; H^{s}\right)$, with $s \geqslant 1$ and $s>d / 2$, then

$$
\left\|u-u^{h}\right\|_{L^{\infty}\left([0, T] ; L^{2}\right)} \lesssim h^{s} \quad \text { and } \quad\left\|u-u^{h}\right\|_{L^{\infty}\left([0, T] ; H^{1}\right) \underset{h \rightarrow 0}{\longrightarrow} 0 .} 0 .
$$

If in addition $s>1$, then

$$
\left\|u-u^{h}\right\|_{L^{\infty}\left([0, T] ; H^{1}\right)} \lesssim h^{s-1} .
$$

Remark 4.4. Suppose $u_{0}$ sufficiently smooth. If $\epsilon=+1$ (defocusing case), the bounds for $u$ are known in several cases, with $T>0$ arbitrarily large. On the contrary, if $\epsilon=-1$ (focusing case), $T$ may have to be finite, bounded by a blow-up time. See [Cazenave 2003; Ginibre and Velo 1984]. Typically, if $\sigma=d=1$, then the assumption of the first point is fulfilled for all $T>0$ as soon as $u_{0} \in L^{2}(\mathbb{R})$, for $\epsilon \in\{ \pm 1\}$, from [Tsutsumi 1987], and if $\sigma \geqslant 1, d \leqslant 2$, the assumption of the third point is fulfilled for all $T>0$ as soon as $u_{0} \in H^{s}\left(\mathbb{R}^{d}\right)$, for $\epsilon=+1$, from [Ginibre and Velo 1984].

Proof. For fixed $h>0$, Theorem 2.1 shows that $u^{h} \in C\left(\mathbb{R} ; H^{k}\right)$, with $k=0,1$ or $s$ according to the cases considered in the assumptions of the theorem. Of course, the bounds provided by Theorem 2.1 blow up as $h \rightarrow 0$ if $k>0$.

As in the proof of Proposition 3.1, let $w^{h}=u-u^{h}$. The equation satisfied by $w^{h}$ is simpler than in the proof of Proposition 3.1, since $P_{h}(D)=\Delta$ :

$$
i \partial_{t} w^{h}+\Delta w^{h}=\epsilon\left(\Pi_{h}\left(|u|^{2}\right)\right)^{\sigma} u-\epsilon\left(\Pi_{h}\left(\left|u^{h}\right|^{2}\right)\right)^{\sigma} u^{h}+\epsilon\left(|u|^{2 \sigma}-\left(\Pi_{h}\left(|u|^{2}\right)\right)^{\sigma}\right) u .
$$

We resume the notation

$$
R^{h}(u)=\epsilon\left(|u|^{2 \sigma}-\left(\Pi_{h}\left(|u|^{2}\right)\right)^{\sigma}\right) u \quad \text { and } \quad \Pi_{h}(f)=K_{h} * f,
$$

with $K_{h}(x)=(2 \pi)^{-d / 2} h^{-d} \widehat{\chi}(-x / h)$. From the Young inequality, we have, for all $q \in[1, \infty]$,

$$
\left\|\Pi_{h}(f)\right\|_{L^{q}} \leqslant\left\|K_{h}\right\|_{L^{1}}\|f\|_{L^{q}} \leqslant\|\widehat{\chi}\|_{L^{1}}\|f\|_{L^{q}},
$$

an estimate which is uniform in $h>0$. Introduce the Lebesgue exponents

$$
q=2 \sigma+2, \quad p=\frac{4 \sigma+4}{d \sigma}, \quad \theta=\frac{2 \sigma(2 \sigma+2)}{2-(d-2) \sigma} .
$$

The pair $(p, q)$ is admissible, and

$$
\frac{1}{q^{\prime}}=\frac{2 \sigma}{q}+\frac{1}{q}, \quad \frac{1}{p^{\prime}}=\frac{2 \sigma}{\theta}+\frac{1}{p} .
$$


For $t>0$, write $L_{t}^{j} L^{k}=L^{j}\left([0, t] ; L^{k}\left(\mathbb{R}^{d}\right)\right)$. From the Strichartz estimates (see [Cazenave 2003]),

$$
\begin{aligned}
\left\|w^{h}\right\|_{L_{t}^{p} L^{q} \cap L_{t}^{\infty} L^{2}} & \lesssim\left\|\left(\Pi_{h}\left(|u|^{2}\right)\right)^{\sigma} u-\left(\Pi_{h}\left(\left|u^{h}\right|^{2}\right)\right)^{\sigma} u^{h}\right\|_{L_{t}^{p^{\prime}} L^{q^{\prime}}}+\left\|R^{h}(u)\right\|_{L_{t}^{p_{1}^{\prime}} L^{q_{1}^{\prime}}} \\
& \lesssim\left(\|u\|_{L_{t}^{\theta} L^{q}}^{2 \sigma}+\left\|u^{h}\right\|_{L_{t}^{\theta} L^{q}}^{2 \sigma}\left\|w^{h}\right\|_{L_{t}^{p} L^{q}}+\left\|R^{h}(u)\right\|_{L_{t}^{p_{1}^{\prime}} L^{q_{1}^{\prime}}},\right.
\end{aligned}
$$

where we have used the Hölder inequality and (4-1), and where $\left(p_{1}, q_{1}\right)$ is an admissible pair whose value will be given later.

If $\sigma=1$ and $d \leqslant 2$, then $\theta \leqslant p$, and we infer

$$
\left\|w^{h}\right\|_{L_{t}^{p} L^{q} \cap L_{t}^{\infty} L^{2}} \lesssim t^{1 / \theta-1 / p}\left(\|u\|_{L_{t}^{p} L^{q}}^{2 \sigma}+\left\|u^{h}\right\|_{L_{t}^{p} L^{q}}^{2 \sigma}\right)\left\|w^{h}\right\|_{L_{t}^{p} L^{q}}+\left\|R^{h}(u)\right\|_{L_{t}^{p_{1}^{\prime}} L^{q_{1}^{\prime}}} .
$$

In the first case of the theorem, we assume $u \in L^{p}\left([0, T] ; L^{q}\right)$, since $p=8 / d$ and $q=4$ for $\sigma=1$. We use again a bootstrap argument: so long as $\left\|u^{h}\right\|_{L_{t}^{p} L^{q}} \leqslant 2\|u\|_{L_{t}^{p} L^{q}}$, we divide the interval $[0, T]$ into finitely many small intervals so the first term of the right hand side is absorbed by the left hand side (recall that $p$ is finite), and we have

$$
\left\|w^{h}\right\|_{L_{t}^{p} L^{q} \cap L_{t}^{\infty} L^{2}} \lesssim\left\|R^{h}(u)\right\|_{L_{t}^{p_{1}^{\prime}} L_{1}^{q_{1}^{\prime}}} .
$$

The bootstrap argument is validated provided that $\left\|R^{h}(u)\right\|_{L_{T}^{p_{1}^{\prime}} L^{q_{1}^{\prime}}} \rightarrow 0$ as $h \rightarrow 0$.

If we have only $\sigma<2 /(d-2)$, then by the Sobolev embedding,

$$
\|u\|_{L_{t}^{\theta} L^{q}} \leqslant t^{1 / \theta}\|u\|_{L_{t}^{\infty} H^{1}} .
$$

In the same way as above,

$$
\left\|\nabla w^{h}\right\|_{L_{t}^{p} L^{q} \cap L_{t}^{\infty} L^{2}} \lesssim\left\|\nabla\left(\left(\Pi_{h}\left(|u|^{2}\right)\right)^{\sigma} u-\left(\Pi_{h}\left(\left|u^{h}\right|^{2}\right)\right)^{\sigma} u^{h}\right)\right\|_{L_{t}^{p^{\prime}} L^{q^{\prime}}}+\left\|\nabla R^{h}(u)\right\|_{L_{t}^{p_{1}^{\prime}} L^{q_{1}^{\prime}}}
$$

The first term of the right hand side is controlled by

$$
\left\|\left(\Pi_{h}\left(|u|^{2}\right)\right)^{\sigma} \nabla u-\left(\Pi_{h}\left(\left|u^{h}\right|^{2}\right)\right)^{\sigma} \nabla u^{h}\right\|_{L_{t}^{p^{\prime}} L^{q^{\prime}}}+\left\|u \nabla\left(\Pi_{h}\left(|u|^{2}\right)\right)^{\sigma}-u^{h} \nabla\left(\Pi_{h}\left(\left|u^{h}\right|^{2}\right)\right)^{\sigma}\right\|_{L_{t}^{p^{\prime}} L^{q^{\prime}}}
$$

Introducing the factor $\left(\Pi_{h}\left(|u|^{2}\right)\right)^{\sigma} \nabla u^{h}$, the first term is estimated by

$$
\begin{aligned}
\left\|\left(\Pi_{h}\left(|u|^{2}\right)\right)^{\sigma} \nabla w^{h}\right\|_{L_{t}^{p^{\prime}} L^{q^{\prime}}}+\left\|\left(\left(\Pi_{h}\left(|u|^{2}\right)\right)^{\sigma}-\left(\Pi_{h}\left(\left|u^{h}\right|^{2}\right)\right)^{\sigma}\right) \nabla u^{h}\right\|_{L_{t}^{p^{\prime}} L^{q^{\prime}}} \\
\quad \lesssim\left\|\Pi_{h}\left(|u|^{2}\right)\right\|_{L_{t}^{\theta / 2} L^{q / 2}}^{\sigma}\left\|\nabla w^{h}\right\|_{L_{t}^{p} L^{q}}+\left(\|u\|_{L_{t}^{\theta} L^{q}}^{2 \sigma-2}+\left\|u^{h}\right\|_{L_{t}^{\theta} L^{q}}^{2 \sigma-2}\right)\left\||u|^{2}-\left|u^{h}\right|^{2}\right\|_{L_{t}^{\theta / 2} L^{q / 2}}\left\|\nabla u^{h}\right\|_{L_{t}^{p} L^{q}} \\
\quad \lesssim\|u\|_{L_{t}^{\theta} L^{q}}^{2 \sigma}\left\|\nabla w^{h}\right\|_{L_{t}^{p} L^{q}}+\left(\|u\|_{L_{t}^{\theta} L^{q}}^{2 \sigma-1}+\left\|u^{h}\right\|_{L_{t}^{\theta} L^{q}}^{2 \sigma-1}\right)\left\|w^{h}\right\|_{L_{t}^{\theta} L^{q}}\left\|\nabla u^{h}\right\|_{L_{t}^{p} L^{q}} \\
\quad \lesssim t^{2 \sigma / \theta}\|u\|_{L_{t}^{\infty} H^{1}}^{2 \sigma}\left\|\nabla w^{h}\right\|_{L_{t}^{p} L^{q}}+t^{2 \sigma / \theta}\left(\|u\|_{L_{t}^{\infty} H^{1}}^{2 \sigma-1}+\left\|u^{h}\right\|_{L_{t}^{\infty} H^{1}}^{2 \sigma-1}\right)\left\|w^{h}\right\|_{L_{t}^{\infty} H^{1}}\left\|\nabla u^{h}\right\|_{L_{t}^{p} L^{q}} .
\end{aligned}
$$

Proceeding similarly for the other term in (4-3), splitting $[0, T]$ into finitely many time intervals where the terms containing $w^{h}$ on the right hand side can be absorbed by the left hand side, and using a bootstrap argument, we end up with

$$
\left\|w^{h}\right\|_{L_{t}^{p} W^{1, q} \cap L_{t}^{\infty} H^{1}} \lesssim\left\|R^{h}(u)\right\|_{L_{t}^{p_{1}^{\prime}} W^{1, q_{1}^{\prime}}}
$$


Therefore, it suffices to show that for some admissible pair $\left(p_{1}, q_{1}\right)$, the source term converges to 0 in $L^{p_{1}^{\prime}}\left([0, T] ; L^{q_{1}^{\prime}}\right)$ (if $\sigma=1$ and $d \leqslant 2$ ) or in $L^{p_{1}^{\prime}}\left([0, T] ; W^{1, q_{1}^{\prime}}\right)$ (in the other cases), so the bootstrap argument is completed. In addition, the rate of converge of the source term, if any, yields a rate of convergence for $w^{h}$. The theorem then stems from the following lemma, in which $(p, q)$ is given by (4-2).

Lemma 4.5. Let $T>0$. The source term $R^{h}(u)$ can be controlled as follows.

1. Suppose that $\sigma=1$ and $d \leqslant 2$. If $u \in L^{\infty}\left([0, T] ; L^{2}\right) \cap L^{8 / d}\left([0, T] ; L^{4}\right)$, then

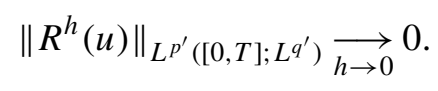

2. Suppose that $\sigma=1$ and $d=3$.

- If $u, \nabla u \in L^{\infty}\left([0, T] ; L^{2}\right) \cap L^{8 / d}\left([0, T] ; L^{4}\right)$, then

$$
\left\|R^{h}(u)\right\|_{L^{p^{\prime}}\left([0, T] ; W^{1, q^{\prime}}\right)}^{\underset{h \rightarrow 0}{\longrightarrow} 0 .}
$$

- If $u \in L^{\infty}\left([0, T] ; H^{s}\right)$, with $s>3 / 2$, then

$$
\left\|R^{h}(u)\right\|_{L^{1}\left([0, T] ; L^{2}\right)} \lesssim h^{s} \quad \text { and } \quad\left\|R^{h}(u)\right\|_{L^{1}\left([0, T] ; H^{1}\right)} \lesssim h^{s-1} .
$$

3. Suppose that $\sigma \geqslant 1$ and $d \leqslant 2$. If $u \in L^{\infty}\left([0, T] ; H^{s}\right)$, with $s \geqslant 1$ and $s>d / 2$, then

$$
\left\|R^{h}(u)\right\|_{L^{1}\left([0, T] ; L^{2}\right)} \lesssim h^{s} \quad \text { and } \quad\left\|R^{h}(u)\right\|_{L^{1}\left([0, T] ; H^{1}\right)} \underset{h \rightarrow 0}{\longrightarrow} 0 .
$$

If in addition $s>1$, then

$$
\left\|R^{h}(u)\right\|_{L^{1}\left([0, T] ; H^{1}\right)} \lesssim h^{s-1} .
$$

Proof of Lemma 4.5. For the first case, we use the Hölder inequality, in view of (4-2):

$$
\left\|R^{h}(u)\right\|_{L_{T}^{p^{\prime}} L^{q^{\prime}}}=\left\|\left(1-\Pi_{h}\right)\left(|u|^{2}\right) u\right\|_{L_{T}^{p^{\prime}} L^{q^{\prime}}} \leqslant\left\|\left(1-\Pi_{h}\right)\left(|u|^{2}\right)\right\|_{L_{T}^{\theta / 2} L^{q / 2}}\|u\|_{L_{T}^{p} L^{q}} .
$$

We note that for $\sigma=1, q=4$, so by the Plancherel theorem,

$$
\left\|\left(1-\Pi_{h}\right)\left(|u|^{2}\right)\right\|_{L^{2}}^{2}=\int_{\mathbb{R}^{d}}(1-\chi(h \xi))^{2}\left|\mathscr{F}\left(|u|^{2}\right)(\xi)\right|^{2} d \xi \leqslant \int_{|\xi|>1 / h}\left|\mathscr{F}\left(|u|^{2}\right)(\xi)\right|^{2} d \xi .
$$

By assumption, $u \in L^{p}\left([0, T] ; L^{4}\right) \subset L^{\theta}\left([0, T] ; L^{4}\right)$, thus $|u|^{2} \in L^{\theta / 2}\left([0, T] ; L^{2}\right)$, and by the Plancherel theorem, $\mathscr{F}\left(|u|^{2}\right) \in L^{\theta / 2}\left([0, T] ; L^{2}\right)$. The first point of the lemma then stems from the dominated convergence theorem.

For the first case of the second point, we note that now $\theta>p$, so the above argument must be adapted, and we have to estimate the gradient of $R^{h}(u)$ in the same space as above. Since we have $L^{\infty}\left([0, T] ; H^{1}\left(\mathbb{R}^{3}\right)\right) \subset L^{\theta}\left([0, T] ; L^{4}\left(\mathbb{R}^{3}\right)\right)$, the dominated convergence theorem yields

$$
\left\|R^{h}(u)\right\|_{L_{T}^{p^{\prime}} L^{q^{\prime}}} \underset{h \rightarrow 0}{\longrightarrow} 0 .
$$


We now estimate $\nabla R^{h}(u)$. Write

$$
\begin{aligned}
\left\|\nabla R^{h}(u)\right\|_{L_{T}^{p^{\prime}} L^{q^{\prime}}} & \leqslant\left\|\left(1-\Pi_{h}\right)\left(|u|^{2}\right)\right\|_{L_{T}^{\theta / 2} L^{2}}\|\nabla u\|_{L_{T}^{p} L^{2}}+\left\|\left(1-\Pi_{h}\right) \nabla\left(|u|^{2}\right)\right\|_{L_{T}^{(1 / \theta+1 / p)^{-1} L^{2}}}\|u\|_{L_{T}^{\theta} L^{2}} \\
& \lesssim\left\|\left(1-\Pi_{h}\right)\left(|u|^{2}\right)\right\|_{L_{T}^{\infty} L^{2}}\|\nabla u\|_{L_{T}^{p} L^{2}}+\left\|\left(1-\Pi_{h}\right) \nabla\left(|u|^{2}\right)\right\|_{L_{T}^{(1 / \theta+1 / p)^{-1}} L^{2}}\|u\|_{L_{T}^{\infty} L^{2} .}
\end{aligned}
$$

By the same argument as above,

$$
\left\|\left(1-\Pi_{h}\right)\left(|u|^{2}\right)\right\|_{L_{T}^{\infty} L^{2}}\|\nabla u\|_{L_{T}^{p} L^{2} \underset{h \rightarrow 0}{\longrightarrow} 0 .} 0 .
$$

We note that $u$ bounded in $L^{\infty}\left([0, T] ; H^{1}\left(\mathbb{R}^{3}\right)\right) \subset L^{\theta}\left([0, T] ; L^{4}\left(\mathbb{R}^{3}\right)\right)$, and $\nabla u$ bounded in $L_{T}^{p} L^{4}$, so $\nabla|u|^{2}$ is bounded in $L_{T}^{(1 / \theta+1 / p)^{-1}} L^{2}$. Invoking Plancherel theorem and the dominated convergence theorem like above, we infer that

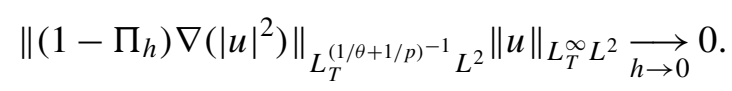

This completes the proof for the first case of the second point.

For the remaining cases, we use that $H^{s}\left(\mathbb{R}^{d}\right)$ is embedded into $L^{\infty}\left(\mathbb{R}^{d}\right)$ : for fixed $t$,

$$
\begin{aligned}
\left\|R^{h}(u)(t)\right\|_{L^{2}} & \lesssim\left(\|u(t)\|_{L^{\infty}}^{2 \sigma-2}+\left\|\Pi_{h}\left(|u(t)|^{2}\right)\right\|_{L^{\infty}}^{\sigma-1}\right)\left\|\left(1-\Pi_{h}\right)\left(|u(t)|^{2}\right)\right\|_{L^{2}}\|u(t)\|_{L^{\infty}} \\
& \lesssim\|u(t)\|_{L^{\infty}}^{2 \sigma-1}\left\|\left(1-\Pi_{h}\right)\left(|u(t)|^{2}\right)\right\|_{L^{2}} \lesssim\|u(t)\|_{H^{s}}^{2 \sigma-1}\left\|\left(1-\Pi_{h}\right)\left(|u(t)|^{2}\right)\right\|_{L^{2}}
\end{aligned}
$$

Like in the proof of Proposition 3.1, we use the estimate

$$
\left\|\left(1-\Pi_{h}\right) f\right\|_{L^{2}} \leqslant h^{s}\|f\|_{H^{s}}
$$

and since $H^{s}\left(\mathbb{R}^{d}\right)$ is an algebra,

$$
\left\|R^{h}(u)\right\|_{L^{\infty}\left([0, T] ; L^{2}\right)} \lesssim h^{s}\|u\|_{L^{\infty}\left([0, T] ; H^{s}\right)}^{2 \sigma+1} .
$$

To conclude the proof, we estimate $\nabla R^{h}(u)$ in $L^{2}\left(\mathbb{R}^{d}\right)$. We compute

$$
\begin{aligned}
\nabla R^{h}(u)=\sigma|u|^{2 \sigma-2}\left(\left(1-\Pi_{h}\right)\left(\nabla\left(|u|^{2}\right)\right)\right) u+\left(|u|^{2 \sigma}-\left(\Pi_{h}\left(|u|^{2}\right)\right)^{\sigma}\right) \nabla u & \\
& +\sigma\left(|u|^{2 \sigma-2}-\left(\Pi_{h}\left(|u|^{2}\right)\right)^{\sigma-1}\right) \Pi_{h}\left(\nabla\left(|u|^{2}\right)\right) u
\end{aligned}
$$

where the second line is zero if $\sigma=1$. We estimate successively, thanks to (4-1),

$$
\begin{aligned}
\left\||u|^{2 \sigma-2}\left(\left(1-\Pi_{h}\right)\left(\nabla\left(|u|^{2}\right)\right)\right) u\right\|_{L^{2}} & \leqslant\|u\|_{L^{\infty}}^{2 \sigma-1}\left\|\left(1-\Pi_{h}\right)\left(|u|^{2}\right)\right\|_{H^{1}} \\
\left\|\left(|u|^{2 \sigma}-\left(\Pi_{h}\left(|u|^{2}\right)\right)^{\sigma}\right) \nabla u\right\|_{L^{2}} & \leqslant\|u\|_{L^{\infty}}^{2 \sigma-2}\left\|\left(1-\Pi_{h}\right)\left(|u|^{2}\right)\right\|_{L^{\infty}}\|\nabla u\|_{L^{2}}
\end{aligned}
$$

and, if $\sigma \geqslant 2$,

$$
\begin{aligned}
\left\|\left(|u|^{2 \sigma-2}-\left(\Pi_{h}\left(|u|^{2}\right)\right)^{\sigma-1}\right) \Pi_{h}\left(\nabla\left(|u|^{2}\right)\right) u\right\|_{L^{2}} & \lesssim\|u\|_{L^{\infty}}^{2 \sigma-4}\left\|\left(1-\Pi_{h}\right)\left(|u|^{2}\right)\right\|_{L^{2}}\left\|\nabla\left(|u|^{2}\right)\right\|_{L^{2}}\|u\|_{L^{\infty}} \\
& \lesssim\|u\|_{L^{\infty}}^{2 \sigma-2}\left\|\left(1-\Pi_{h}\right)\left(|u|^{2}\right)\right\|_{L^{2}}\|\nabla u\|_{L^{2}} .
\end{aligned}
$$

Since we have $H^{s}\left(\mathbb{R}^{d}\right) \hookrightarrow L^{\infty}\left(\mathbb{R}^{d}\right)$, we end up with

$$
\left\|\nabla R^{h}(u)\right\|_{L^{2}} \lesssim\|u\|_{H^{s}}^{2 \sigma-2}\left\|\left(1-\Pi_{h}\right)\left(|u|^{2}\right)\right\|_{H^{1}} .
$$


If $s>1$, (4-4) yields, since in addition $s>d / 2$,

$$
\left\|\left(1-\Pi_{h}\right)\left(|u|^{2}\right)\right\|_{H^{1}} \lesssim h^{s-1}\left\||u|^{2}\right\|_{H^{s}} \lesssim h^{s-1}\|u\|_{H^{s}}^{2} .
$$

If $s=1$ (a case which may occur only if $d=1$, since $s>d / 2$ ), we write

$$
\left\|\nabla\left(1-\Pi_{h}\right)\left(|u|^{2}\right)\right\|_{L^{2}}^{2} \leqslant \int_{|\xi|>1 / h}\left|\mathscr{F}\left(\nabla\left(|u|^{2}\right)\right)(\xi)\right|^{2} d \xi
$$

Now since $\nabla\left(|u|^{2}\right)=2 \operatorname{Re} \bar{u} \nabla u$ and $u \in H^{1}(\mathbb{R}) \hookrightarrow L^{\infty}(\mathbb{R}), \nabla u \in L^{2}(\mathbb{R})$, we conclude thanks to the dominated convergence theorem.

This completes the proof of Theorem 4.3, by choosing $\left(p_{1}, q_{1}\right)=(p, q)$ or $(\infty, 2)$.

\section{Appendix: Physical saturation of the nonlinearity}

Instead of cutting off the high frequencies, one may be tempted to saturate the nonlinear potential, by replacing $|u|^{2}$ not by $\Pi\left(|u|^{2}\right)$ but by $f\left(|u|^{2}\right)$ where $f$ is smooth, equal to the identity near the origin, and constant at infinity. Note also that a saturated nonlinearity may be in better agreement with physical models (recall however that (1-1) appears in rather different physical contexts, such as quantum mechanics, optics, and even fluid mechanics), since typically the power-like nonlinearity in (1-1) may stem from a Taylor expansion; see [Lannes 2011; Sulem and Sulem 1999]. More precisely, let $f \in C^{\infty}(\mathbb{R} ; \mathbb{R})$ such that

$$
f(s)= \begin{cases}s & \text { if } 0 \leqslant s \leqslant 1 \\ 2 & \text { if } s \geqslant 2\end{cases}
$$

The analogue of the Fourier multiplier $\Pi_{h}$ is defined as

$$
f_{h}\left(|u|^{2}\right)=\frac{1}{h} f\left(h|u|^{2}\right),
$$

and we replace (2-5) with

$$
i \partial_{t} u^{h}+P_{h}(D) u^{h}=\epsilon\left(f_{h}\left(\left|u^{h}\right|^{2}\right)\right)^{\sigma} u^{h},
$$

so the formal conservation of the $L^{2}$-norm still holds. We could also consider

$$
f_{h}\left(|u|^{2}\right)=\frac{|u|^{2}}{1+h|u|^{2}} .
$$

In both cases, the main aspect to notice is that $f_{h}$ is bounded and $z \mapsto f_{h}\left(|z|^{2}\right)^{\sigma} z$ is globally Lipschitzian. We infer the analogue of Theorem 2.1, at least in the $L^{2}$ case.

Proposition A.1. Let $\sigma \in \mathbb{N}, \epsilon \in\{ \pm 1\}, P: \mathbb{R}^{d} \rightarrow \mathbb{R}$ and $f$ given either by (A-1) or by (A-3).

- For any $u_{0} \in L^{2}\left(\mathbb{R}^{d}\right)$, (A-2) has a unique solution $u^{h} \in C\left(\mathbb{R} ; L^{2}\left(\mathbb{R}^{d}\right)\right)$ such that $u_{\mid t=0}^{h}=u_{0}$. Its $L^{2}$-norm is independent of time. 
- The flow map $u_{0} \mapsto u^{h}$ is uniformly continuous from the balls in $L^{2}\left(\mathbb{R}^{d}\right)$ to $C\left(\mathbb{R} ; L^{2}\left(\mathbb{R}^{d}\right)\right)$. More precisely, for all $u_{0}, v_{0} \in L^{2}\left(\mathbb{R}^{d}\right)$, there exists $C$ depending on $\sigma, h,\left\|u_{0}\right\|_{L^{2}}$ and $\left\|v_{0}\right\|_{L^{2}}$ such that for all $T>0$,

$$
\left\|u^{h}-v^{h}\right\|_{L^{\infty}\left([-T, T] ; L^{2}\left(\mathbb{R}^{d}\right)\right)} \leqslant\left\|u_{0}-v_{0}\right\|_{L^{2}\left(\mathbb{R}^{d}\right)} e^{C T},
$$

where $u^{h}$ and $v^{h}$ denote the solutions to (A-2) with data $u_{0}$ and $v_{0}$, respectively.

Introduce

$$
F_{h}(s)=\int_{0}^{s} f_{h}(y)^{\sigma} d y .
$$

We check that the following conservation of energy holds:

$$
\frac{d}{d t}\left(\int_{\mathbb{R}^{d}} \bar{u}^{h}(t, x) P_{h}(D) u^{h}(t, x) d x+\epsilon \int_{\mathbb{R}^{d}} F_{h}\left(|u(t, x)|^{2}\right) d x\right)=0 .
$$

Proving the analogue of Proposition 3.1 is easy in the case (A-1), since the last source term for the error $w^{h}$ is now

$$
R^{h}(u)=\left(|u|^{2 \sigma}-f_{h}\left(|u|^{2}\right)^{\sigma}\right) u
$$

and under the assumptions of Proposition $3.1, u \in L^{\infty}\left([0, T] \times \mathbb{R}^{d}\right)$, so there exists $h_{0}>0$ such that for $0<h \leqslant h_{0}$,

$$
|u(t, x)|^{2 \sigma}=f_{h}\left(|u(t, x)|^{2}\right)^{\sigma} \quad \text { for all }(t, x) \in[0, T] \times \mathbb{R}^{d} .
$$

Therefore, this source term simply vanishes for $h$ sufficiently small. In the case (A-3), we can use the relation

$$
\left|R_{h}(u)\right|=\left|\left(|u|^{2 \sigma}-f_{h}\left(|u|^{2}\right)^{\sigma}\right) u\right| \lesssim \frac{h|u|^{2}}{1+h|u|^{2}}|u|^{2 \sigma+1}
$$

and the Schauder lemma to get a source term which is $O(h)$ in $H^{s}\left(\mathbb{R}^{d}\right)$, for $s>d / 2$.

Proposition A.2. Let $\sigma \in \mathbb{N}$. We assume that $P$ is such that $P_{h}(\xi)=-|\xi|^{2}+\mathcal{O}\left(h^{\alpha}\langle\xi\rangle^{\beta}\right)$ for some $\alpha, \beta \geqslant 0$. Denote by $u^{h}$ the solution to (A-2) with $P_{h}$ and $f_{h}$, such that $u_{\mid t=0}^{h}=u_{\mid t=0}$. Suppose that the solution to (1-1) satisfies $u \in L^{\infty}\left([0, T] ; H^{s+\beta}\right)$, for some $s>d / 2$.

- In the case (A-1), $\left\|u-u^{h}\right\|_{L^{\infty}\left([0, T] ; H^{s}\right)} \lesssim h^{\alpha}$.

- In the case (A-3), $\left\|u-u^{h}\right\|_{L^{\infty}\left([0, T] ; H^{s}\right)} \lesssim h^{\min (\alpha, 1)}$.

In the case (A-1), proving an analogue to Theorem 4.3 seems to be more delicate though, and we choose not to investigate this aspect here. On the other hand, in the case (A-3), using the estimate (A-4), Strichartz estimates and Hölder inequalities with the "standard" Lebesgue exponents (in the same fashion as in the proof of Theorem 4.3, see [Cazenave 2003]), we have, with steps similar to those presented in the proof of Theorem 4.3:

Theorem A.3. Let $\sigma \in \mathbb{N}$ and $T>0$. Let $u$ solve (1-1), and consider a solution $u^{h}$ to

$$
i \partial_{t} u^{h}+\Delta u^{h}=\epsilon\left(\frac{\left|u^{h}\right|^{2}}{1+h\left|u^{h}\right|^{2}}\right)^{\sigma} u^{h}, \quad u_{\mid t=0}^{h}=u_{0} .
$$


1. If $\sigma \leqslant 2 / d$, and $u \in L^{\infty}\left([0, T] ; L^{2}\right) \cap L^{(4 \sigma+4) / d \sigma}\left([0, T] ; L^{2 \sigma+2}\right)$, then

$$
\left\|u-u^{h}\right\|_{L^{\infty}\left([0, T] ; L^{2}\right)} \underset{h \rightarrow 0}{\longrightarrow} 0 .
$$

2. Suppose that $\sigma=1$ and $d=3$.

- If $u, \nabla u \in L^{\infty}\left([0, T] ; L^{2}\right) \cap L^{8 / d}\left([0, T] ; L^{4}\right)$, then

$$
\left\|u-u^{h}\right\|_{L^{\infty}\left([0, T] ; H^{1}\right)} \underset{h \rightarrow 0}{\longrightarrow} 0 .
$$

- If $u \in L^{\infty}\left([0, T] ; H^{s}\right)$, with $s>3 / 2$, then

$$
\left\|u-u^{h}\right\|_{L^{\infty}\left([0, T] ; H^{1}\right)} \lesssim h .
$$

3. Suppose that $\sigma \geqslant 1$ and $d \leqslant 2$. If $u \in L^{\infty}\left([0, T] ; H^{s}\right)$, with $s \geqslant 1$ and $s>d / 2$, then

$$
\left\|u-u^{h}\right\|_{L^{\infty}\left([0, T] ; H^{1}\right)} \lesssim h .
$$

\section{References}

[Alazard and Carles 2009] T. Alazard and R. Carles, "Loss of regularity for supercritical nonlinear Schrödinger equations", Math. Ann. 343:2 (2009), 397-420. MR 2009j:35341 Zbl 1161.35047

[Bejenaru and Tao 2006] I. Bejenaru and T. Tao, "Sharp well-posedness and ill-posedness results for a quadratic non-linear Schrödinger equation”, J. Funct. Anal. 233:1 (2006), 228-259. MR 2007i:35216 Zbl 1090.35162

[Burq 2011] N. Burq, "Large-time dynamics for the one-dimensional Schrödinger equation", Proc. Roy. Soc. Edinburgh Sect. A 141:2 (2011), 227-251. MR 2012f:35499 Zbl 1226.35072

[Burq and Tzvetkov 2008a] N. Burq and N. Tzvetkov, "Random data Cauchy theory for supercritical wave equations. I. Local theory", Invent. Math. 173:3 (2008), 449-475. MR 2009k:58057 Zbl 1156.35062

[Burq and Tzvetkov 2008b] N. Burq and N. Tzvetkov, "Random data Cauchy theory for supercritical wave equations. II. A global existence result”, Invent. Math. 173:3 (2008), 477-496. MR 2010i:58025 Zbl 1187.35233

[Burq et al. 2005] N. Burq, P. Gérard, and N. Tzvetkov, "Multilinear eigenfunction estimates and global existence for the three dimensional nonlinear Schrödinger equations", Ann. Sci. École Norm. Sup. (4) 38:2 (2005), 255-301. MR 2006m:35337 Zbl 1116.35109

[Burq et al. 2012] N. Burq, L. Thomann, and N. Tzvetkov, "Long time dynamics for the one dimensional non linear Schrödinger equation", preprint, 2012. To appear in Ann. Inst. Fourier. arXiv 1002.4054

[Carles 2007] R. Carles, "Geometric optics and instability for semi-classical Schrödinger equations", Arch. Ration. Mech. Anal. 183:3 (2007), 525-553. MR 2007j:35211 Zbl 1134.35098

[Carles 2011] R. Carles, "On Schrödinger equations with modified dispersion", Dyn. Partial Differ. Equ. 8:3 (2011), 173-183. MR 2901602 Zbl 06079139

[Carles et al. 2012] R. Carles, E. Dumas, and C. Sparber, "Geometric optics and instability for NLS and Davey-Stewartson models", J. Eur. Math. Soc. 14:6 (2012), 1885-1921. MR 2984591

[Cazenave 2003] T. Cazenave, Semilinear Schrödinger equations, Courant Lecture Notes in Mathematics 10, Courant Institute of Mathematical Sciences, New York, 2003. MR 2004j:35266 Zbl 1055.35003

[Cazenave and Weissler 1990] T. Cazenave and F. B. Weissler, "The Cauchy problem for the critical nonlinear Schrödinger equation in $H^{s ", ~ N o n l i n e a r ~ A n a l . ~ 14: 10 ~(1990), ~ 807-836 . ~ M R ~ 91 j: 35252 ~ Z b l ~} 0706.35127$

[Cazenave et al. 2011] T. Cazenave, D. Fang, and Z. Han, "Continuous dependence for NLS in fractional order spaces", Ann. Inst. H. Poincaré Anal. Non Linéaire 28:1 (2011), 135-147. MR 2012a:35292 Zbl 1209.35124

[Christ et al. 2003] M. Christ, J. Colliander, and T. Tao, "Ill-posedness for nonlinear Schrödinger and wave equations", preprint, 2003. arXiv math/0311048 
[Colliander et al. 2002] J. Colliander, M. Keel, G. Staffilani, H. Takaoka, and T. Tao, "Almost conservation laws and global rough solutions to a nonlinear Schrödinger equation”, Math. Res. Lett. 9:5-6 (2002), 659-682. MR 2003j:35294 Zbl 1152.35491

[Debussche and Faou 2009] A. Debussche and E. Faou, "Modified energy for split-step methods applied to the linear Schrödinger equation”, SIAM J. Numer. Anal. 47:5 (2009), 3705-3719. MR 2010m:65169 Zbl 1209.65137

[Ginibre and Velo 1984] J. Ginibre and G. Velo, "On the global Cauchy problem for some nonlinear Schrödinger equations", Ann. Inst. H. Poincaré Anal. Non Linéaire 1:4 (1984), 309-323. MR 87a:35164 Zbl 0569.35070

[Ignat and Zuazua 2012] L. I. Ignat and E. Zuazua, "Convergence rates for dispersive approximation schemes to nonlinear Schrödinger equations”, J. Math. Pures Appl. (9) 98:5 (2012), 479-517. MR 2980459

[Kato 1987] T. Kato, “On nonlinear Schrödinger equations”, Ann. Inst. H. Poincaré Phys. Théor. 46:1 (1987), 113-129. MR 88f:35133 Zbl 0632.35038

[Kenig et al. 2001] C. E. Kenig, G. Ponce, and L. Vega, "On the ill-posedness of some canonical dispersive equations", Duke Math. J. 106:3 (2001), 617-633. MR 2002c:35265 Zbl 1034.35145

[Lannes 2011] D. Lannes, "High-frequency nonlinear optics: from the nonlinear Schrödinger approximation to ultrashort-pulses equations", Proc. Roy. Soc. Edinburgh Sect. A 141:2 (2011), 253-286. MR 2012i:35221 Zb1 1219.35283

[Lebeau 2001] G. Lebeau, "Non linear optic and supercritical wave equation”, Bull. Soc. Roy. Sci. Liège 70:4-6 (2001), $267-306$. MR 2003b:35202 Zbl 1034.35137

[Lebeau 2005] G. Lebeau, "Perte de régularité pour les équations d'ondes sur-critiques”, Bull. Soc. Math. France 133:1 (2005), 145-157. MR 2006c:35194 Zbl 1071.35020

[Métivier 2004] G. Métivier, "Exemples d'instabilités pour des équations d'ondes non linéaires (d'après G. Lebeau)", pp. vii, 63-75 in Séminaire Bourbaki 2002/2003 (Exposé 911), Astérisque 294, Société Mathématique de France, Paris, 2004. MR 2005k:35287 Zbl 1215.35106

[Sulem and Sulem 1999] C. Sulem and P.-L. Sulem, The nonlinear Schrödinger equation: Self-focusing and wave collapse, Applied Mathematical Sciences 139, Springer, New York, 1999. MR 2000f:35139 Zbl 0928.35157

[Thomann 2008] L. Thomann, "Instabilities for supercritical Schrödinger equations in analytic manifolds", J. Differential Equations 245:1 (2008), 249-280. MR 2009b:58054 Zbl 1157.35107

[Tsutsumi 1987] Y. Tsutsumi, “ $L^{2}$-solutions for nonlinear Schrödinger equations and nonlinear groups”, Funkcial. Ekvac. 30:1 (1987), 115-125. MR 89c:35143 Zbl 0638.35021

Received 8 Dec 2011. Revised 15 Feb 2012. Accepted 20 Mar 2012.

RÉMI CARLES: Remi.Carles@math.cnrs.fr

CNRS \& Université Montpellier 2, UMR 5149, Mathématiques, CC051, Place Eugène Bataillon, 34095 Montpellier, France 


\title{
Analysis \& PDE
}

\author{
msp.berkeley.edu/apde
}

EDITORS

EDITOR-IN-CHIEF

Maciej Zworski

University of California

Berkeley, USA

BOARD OF EDITORS

\begin{tabular}{|c|c|c|c|}
\hline Michael Aizenman & $\begin{array}{l}\text { Princeton University, USA } \\
\text { aizenman@math.princeton.edu }\end{array}$ & Nicolas Burq & $\begin{array}{l}\text { Université Paris-Sud 11, France } \\
\text { nicolas.burq@math.u-psud.fr }\end{array}$ \\
\hline Luis A. Caffarelli & $\begin{array}{l}\text { University of Texas, USA } \\
\text { caffarel@ math.utexas.edu }\end{array}$ & un-Yung Alice Chang & $\begin{array}{l}\text { Princeton University, USA } \\
\text { chang@ math.princeton.edu }\end{array}$ \\
\hline Michael Christ & $\begin{array}{l}\text { University of California, Berkeley, USA } \\
\text { mchrist@math.berkeley.edu }\end{array}$ & Charles Fefferman & $\begin{array}{l}\text { Princeton University, USA } \\
\text { cf@math.princeton.edu }\end{array}$ \\
\hline Ursula Hamenstaedt & $\begin{array}{l}\text { Universität Bonn, Germany } \\
\text { ursula@math.uni-bonn.de }\end{array}$ & Nigel Higson & $\begin{array}{l}\text { Pennsylvania State Univesity, USA } \\
\text { higson@math.psu.edu }\end{array}$ \\
\hline Vaughan Jones & $\begin{array}{l}\text { University of California, Berkeley, USA } \\
\text { vfr@math.berkeley.edu }\end{array}$ & Herbert Koch & $\begin{array}{l}\text { Universität Bonn, Germany } \\
\text { koch@math.uni-bonn.de }\end{array}$ \\
\hline Izabella Laba & $\begin{array}{l}\text { University of British Columbia, Canada } \\
\text { ilaba@math.ubc.ca }\end{array}$ & Gilles Lebeau & $\begin{array}{l}\text { Université de Nice Sophia Antipolis, France } \\
\text { lebeau@unice.fr }\end{array}$ \\
\hline László Lempert & $\begin{array}{l}\text { Purdue University, USA } \\
\text { lempert@math.purdue.edu }\end{array}$ & Richard B. Melrose & $\begin{array}{l}\text { Massachussets Institute of Technology, USA } \\
\text { rbm@math.mit.edu }\end{array}$ \\
\hline Frank Merle & $\begin{array}{l}\text { Université de Cergy-Pontoise, France } \\
\text { Frank.Merle@u-cergy.fr }\end{array}$ & William Minicozzi II & $\begin{array}{l}\text { Johns Hopkins University, USA } \\
\text { minicozz@ math.jhu.edu }\end{array}$ \\
\hline Werner Müller & $\begin{array}{l}\text { Universität Bonn, Germany } \\
\text { mueller@math.uni-bonn.de }\end{array}$ & Yuval Peres & $\begin{array}{l}\text { University of California, Berkeley, USA } \\
\text { peres@stat.berkeley.edu }\end{array}$ \\
\hline Gilles Pisier & $\begin{array}{l}\text { Texas A\&M University, and Paris } 6 \\
\text { pisier@math.tamu.edu }\end{array}$ & Tristan Rivière & $\begin{array}{l}\text { ETH, Switzerland } \\
\text { riviere@math.ethz.ch }\end{array}$ \\
\hline Igor Rodnianski & $\begin{array}{l}\text { Princeton University, USA } \\
\text { irod@math.princeton.edu }\end{array}$ & Wilhelm Schlag & $\begin{array}{l}\text { University of Chicago, USA } \\
\text { schlag@math.uchicago.edu }\end{array}$ \\
\hline Sylvia Serfaty & $\begin{array}{l}\text { New York University, USA } \\
\text { serfaty@ cims.nyu.edu }\end{array}$ & Yum-Tong Siu & $\begin{array}{l}\text { Harvard University, USA } \\
\text { siu@math.harvard.edu }\end{array}$ \\
\hline Terence Tao & $\begin{array}{l}\text { University of California, Los Angeles, USA } \\
\text { tao@math.ucla.edu }\end{array}$ & Michael E. Taylor & $\begin{array}{l}\text { Univ. of North Carolina, Chapel Hill, USA } \\
\text { met@math.unc.edu }\end{array}$ \\
\hline Gunther Uhlmann & $\begin{array}{l}\text { University of Washington, USA } \\
\text { gunther@math.washington.edu }\end{array}$ & András Vasy & $\begin{array}{l}\text { Stanford University, USA } \\
\text { andras@math.stanford.edu }\end{array}$ \\
\hline an Virgil Voiculescu & $\begin{array}{l}\text { University of California, Berkeley, USA } \\
\text { dvv@ math.berkeley.edu }\end{array}$ & Steven Zelditch & $\begin{array}{l}\text { Northwestern University, USA } \\
\text { zelditch@math.northwestern.edu }\end{array}$ \\
\hline
\end{tabular}

PRODUCTION

production@msp.org

Silvio Levy, Scientific Editor

Sheila Newbery, Senior Production Editor

See inside back cover or msp.berkeley.edu/apde for submission instructions.

The subscription price for 2012 is US \$140/year for the electronic version, and \$240/year for print and electronic. Subscriptions, requests for back issues from the last three years and changes of subscribers address should be sent to Mathematical Sciences Publishers, Department of Mathematics, University of California, Berkeley, CA 94720-3840, USA.

Analysis \& PDE, at Mathematical Sciences Publishers, Department of Mathematics, University of California, Berkeley, CA 94720-3840 is published continuously online. Periodical rate postage paid at Berkeley, CA 94704, and additional mailing offices.

APDE peer review and production are managed by EditFLOW ${ }^{\mathrm{TM}}$ from Mathematical Sciences Publishers.

PUBLISHED BY

mathematical sciences publishers

http://msp.org/

A NON-PROFIT CORPORATION

Typeset in IATEX

Copyright $(2012$ by Mathematical Sciences Publishers 


\section{ANALYSIS \& PDE}

\section{Volume $5 \quad$ No. $5 \quad 2012$}

An inverse problem for the wave equation with one measurement and the pseudorandom 887 source

TAPio Helin, Matti Lassas and LAURi OKSANEN

Two-dimensional nonlinear Schrödinger equation with random radial data YU DENG

Schrödinger operators and the distribution of resonances in sectors

TANYA J. CHRISTIANSEN

Weighted maximal regularity estimates and solvability of nonsmooth elliptic systems, II

PASCAL AUSCHER and ANDREAS RosÉN

The two-phase Stefan problem: regularization near Lipschitz initial data by phase dynamics 1063 SUNHI CHOI and INWON KIM

$C^{\infty}$ spectral rigidity of the ellipse

HAMID HeZARI and STEVE ZELDITCH

A natural lower bound for the size of nodal sets

HAMID HEZARI and CHRISTOPHER D. SOGgE

Effective integrable dynamics for a certain nonlinear wave equation

PATRICK GÉRARD and SANDRINE GRELLIER

Nonlinear Schrödinger equation and frequency saturation 